Nuclear Instruments and Methods 174 (1980) 145-149

() North-Holland Publishing Company

\title{
ON THE FORMATION AND APPLICATION OF LASER INDUCED IONIZATION TRACKS IN GASES
}

\author{
H.J. HILKE \\ CERN, Geneva, Switzerland
}

Received 3.March 1980

It is demonstrated that subnanosecond pulses of a nitrogen laser can produce long ionization tracks in gases, localizable to 50 microns rms in a drift chamber. Primary ionization exceeding 2000 electrons/cm was obtained in $\mathrm{Ar} / \mathrm{CO}_{2}$ and $\mathrm{Ar} / \mathrm{C}_{2} \mathrm{H}_{6} \mathrm{mixtures}$, leading to narrow pulse height spectrum with $9 \% \mathrm{fwhm}$ after gas amplification. The ionization density rises linearly with laser intensity for low powers and approximately exponentially for higher powers. Applications to precision measurements in gas dynamics and related performance studies and calibration of large imaging chambers are discussed. The laser beam technique offers new possibilities especially for measurements in magnetic fields and on double track effects.

\section{Introduction}

The purpose of this investigation was to study the possibility of the creation of long ionization tracks along the beam of an intense ultraviolet laser. The study was stimulated by work on imaging chambers, intended for particle tracking and identification. These chambers * require an accurate knowledge of gas dynamics parameters, like electron drift velocities and drift angles in a magnetic field, diffusion and disturbances from close double tracks. In addition, the actual chamber geometry has to be known to high precision, including wire displacements due to gravity, electrostatic forces and contruction tolerances. Usually, only rather limited information is gained from tests with radioactive sources, from optical surveys and tests in particle beams on small prototypes. The final calibration has to be done on the assembled system in the magnet under data taking conditions. This tends to be a very time consuming iterative process, as it is difficult to separate the various parameters and to obtain clean samples for sufficient statistical analysis.

In this field the laser beam technique looks promising due to its potentially high definition in space and time, and possibly even in amplitude, due to its manoeuvrability and its insensitivity to magnetic fields. Even detailed studies of two track effects

\footnotetext{
* For example the central detectors for the TPC and Mark II at PEP Stanford, Jade at PETRA, Hamburg and AFS and UA1 at CERN, Geneva.
}

might become possible for the first time.

Although the idea has been widely known for some time, little serious research appears to have gone into the subject. The reasons are obvious. Even laser beams were only expected to provide adequate intensities in a tiny volume around the focus of a lens, not over longer distances.

We will present a brief discussion of the theoretical aspects and previous results on laser induced breakdown in gases almost exclusively obtained with strongly focused laser beams. Then, we will describe the experimental set-up and the results and finish with a discussion of some applications of the technique.

\section{Theoretical aspects and previous results}

The ionization potentials of the gases studied, $\mathrm{Ar}$, $\mathrm{CO}_{2}$ and $\mathrm{C}_{2} \mathrm{H}_{6}$ are $15.7,13.7$ and $11.6 \mathrm{eV}$, respectively. The nitrogen laser radiates photons of $3.67 \mathrm{eV}$. Processes to cause ionization would, therefore, be multiphoton processes or field emission. Field emission is expected to require a minimum electric field around $E_{\min }=5 \times 10^{8} \mathrm{~V} / \mathrm{cm}$. The unfocused laser beam, however, might provide maximum fields of $\sim 10^{6} \mathrm{~V} / \mathrm{cm}$. Theoretical estimates for multiphoton processes lead to $E_{\min }=5 \times 10^{7} \mathrm{~V} / \mathrm{cm}$ in $\mathrm{Ar}$ [1]. Experimentally, fifth and seventh order frequency conversion has been observed for fields above $2 \times 10^{8}$ $\mathrm{V} / \mathrm{cm}$ [2]. Other experiments, mostly using ruby laser pulses (1.78 eV, 14-60 ns long), show breakdown in 
Ar at 1 atm in a strongly focused beam for fields

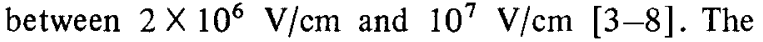
breakdown condition was defined by the production of up to $10^{13}$ ion pairs.

Only recently, laser induced primary ionization has been observed by another group in a drift chamber [9].

Whilst some effects - such as the threshold dependence on pressure and laser frequency (for energies below $2 \mathrm{eV}$ in $\mathrm{Ar}$ ) - agree with the classical theory of diffusion-controlled microwave breakdown, other characteristics remain unexplained: the relatively low breakdown thresholds, the process responsible for the primary ionization and the rapid decrease in breakdown threshold for photon energies above $2 \mathrm{eV}$ in $\mathrm{Ar}$ [6]. This last result, indicating that the breakdown threshold for nitrogen laser frequencies lies a factor of four lower than the threshold for ruby lasers, combined with the ruby laser results [5,7] (thresholds down to $2 \times 10^{6} \mathrm{~V} / \mathrm{cm}$ ) is promising. Indeed, in an unfocused or only weakly focused nitrogen laser beam, we might reach fields not far below $10^{6} \mathrm{~V} / \mathrm{cm}$ over more than a metre.

It should be noted, that the electric fields cited represent only rough estimates of averages. In many experiments subvolumes might have experienced substantially higher fields, e.g. because of multimode excitation.

It seems certain that in most of our experiments, in which the ionization density is found to depend linearly on the laser intensity, multiphoton processes do not play a significant role. Otherwise, our results do not help to clarify the underlying processes; this clearly requires more sophisticated studies. We will, therefore, concentrate on our experimental findings and resulting possibilities for application.

For the above reason our survey of previous experiments was not intended to be complete, as gas breakdown in strongly focused beams is only indirectly linked with our experiments via a possible explanation of the theoretical basis.

At present we cannot completely exclude the possibility that the observed effects are caused by laser induced discharges on microscopic dirt particles. Only conventional precautions for cleaning and filling large wire chambers were applied and commercial grade gases were used with purities of $99.995 \%$ (Ar), 99.9\% $\left(\mathrm{CO}_{2}\right)$ and $99.39 \%\left(\mathrm{C}_{2} \mathrm{H}_{6}\right)$. However, observations like the high density of ion pairs along a track defined in space to 50 microns, the linear dependence of the ionization on laser intensity over a wide range and the small width of the pulse height spectrum let this explanation appear to be very unlikely unless densities $\geqslant 10^{3}$ particles $/ \mathrm{mm}^{3}$ are assumed. On the other hand, gas impurities with low excitation and ionization levels might, via cascade processes, contribute to the low thresholds observed. This question requires further investigation.

\section{Apparatus}

A two-stage nitrogen laser was specially developed for this investigation with the following specifications [10]: light pulse duration $0.5 \mathrm{~ns}$ with a maximum power of $1 \mathrm{MW}$, beam cross section $1 \times 2 \mathrm{~mm}$ and divergence $0.3 \times 0.5 \mathrm{mrad}$. The measured performance was: $<1$ ns duration (using a diode with subnanosecond response [11] limited by the scope rise time), energy burst $100 \mu \mathrm{J}$ (for $2 / 3$ of the design voltage), cross section $1 \times 2 \mathrm{~mm}$, divergence $\sim 0.8 \mathrm{mrad}$. The divergence was estimated from visual inspection of the fluorescence disc on paper. It seemed that most of the light was concentrated in a circle with a diameter well below $1 \mathrm{~mm}$. To estimate an upper limit of the available beam intensity, we assume that a circle with $0.2 \mathrm{~mm}$ diameter receives half the total light. For a pulse width of $0.5 \mathrm{~ns}$ we then obtain a maximum intensity of $J \leqslant 10^{13} \mathrm{~W} / \mathrm{m}^{2}$. From $2 J=$ $\epsilon_{0} c E^{2}$ (in MKS) the maximum electric field becomes: $E_{\max }=10^{6} \mathrm{~V} / \mathrm{cm}$. The pulse repetition rate was set at $4 \mathrm{~Hz}$. A rapid photodiode provided the $t=0$ signal.

The drift chamber, contained in a pressure vessel with two quartz windows, had a drift zone of $22 \mathrm{~mm}$, sense wires of 30 micron diameter spaced at $8 \mathrm{~mm}$ and alternating with potential wires of 100 micron diameter. Care was taken to minimize the material near the sense wire read out; earlier trials had shown that even weak scattered laser light hitting solids nearby can cause pulses bigger than those produced in the gas. The drift chamber could be moved along the laser beam axis and also vertically with respect to it. Vertical displacements would unambiguously differentiate between pulses from ionization in the gas and those originating on solid boundaries. The time delay of the wire signals with respect to the signal from the photodiode would change only for pulses originating in the gas (fig. 1).

To reduce electromagnetic pick-up from the laser discharge in the sensitive amplifier, the drift chamber had to be placed at $4 \mathrm{~m}$ from the laser. Therefore, the use of a lens was required. For most of the work, 


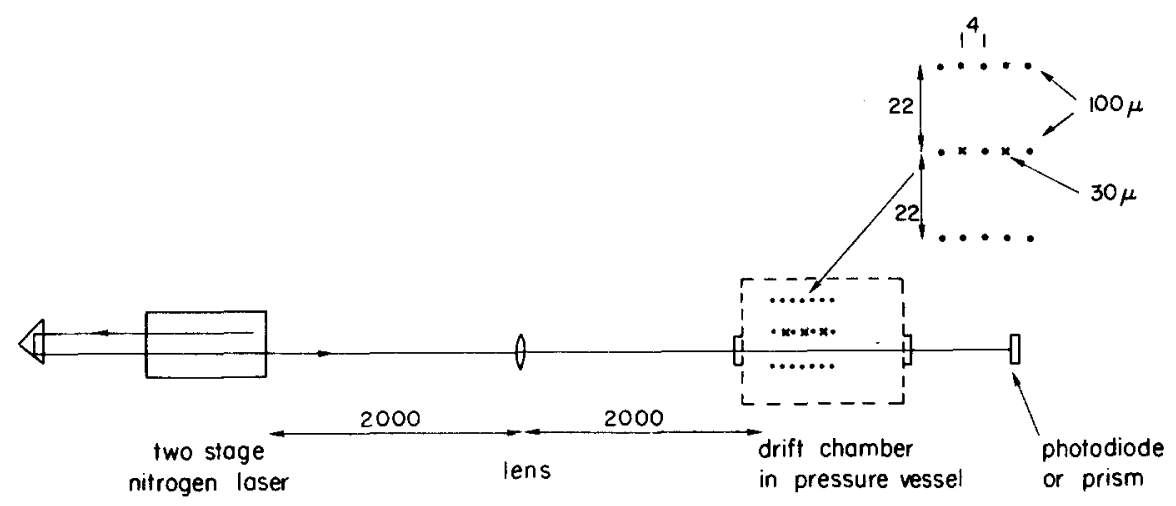

Fig. 1. Schematic view of the apparatus.

lenses with a focal length of $2 \mathrm{~m}$ and $1 \mathrm{~m}$ were used; for the study of higher intensities a focal length of $20 \mathrm{~cm}$ was chosen.

\section{Results}

We tested $\mathrm{Ar} / \mathrm{CO}_{2}$ (45/55 vol. \%) and $\mathrm{Ar} / \mathrm{C}_{2} \mathrm{H}_{6}$ $(50 / 50$ vol. \%) mixtures as typical drift chamber gases, both with satisfying results. The gas pressure was $1.0-1.4 \mathrm{~atm}$, typical drift fields were $1.8 \mathrm{kV} / \mathrm{cm}$ in $\mathrm{Ar} / \mathrm{CO}_{2}$ and $1.1 \mathrm{kV} / \mathrm{cm}$ in $\mathrm{Ar} / \mathrm{C}_{2} \mathrm{H}_{6}$. The potential wire voltage was adjusted to provide a gas amplification of around $10^{4}$.

\subsection{Signal amplitude}

To vary the light intensity, we either used grey filters or changed the focal length or the position of the focus with respect to the sensitive zone. Signals from a pulser and from a $5.9 \mathrm{keV} \gamma$ source $\left({ }^{55} \mathrm{Fe}\right)$ provided calibration of primary ionization and gas amplification.

The signal amplitude was found to rise linearly with light intensity for low powers and approximately exponentially for higher powers. Fig. 2 shows the almost linear regime obtained $3 \mathrm{~cm}$ away from the focus for a $1 \mathrm{~m}$ focal length. At the upper end of the linear region, the laser produced a primary ionization of $\sim 1200$ electrons $/ \mathrm{cm}$.

The pulse height spectrum is asymmetric with a fwhm of $9 \%$ (fig. 3). Inspection on a scope indicated that a major fraction of the amplitude variation was related to laser output variation and that the signal amplitude normalized to the laser output should provide a narrower and possibly more symmetric spectrum.
Primary ionization of more than 100 electrons $/ \mathrm{cm}$ was obtained $60 \mathrm{~cm}$ before and behind the focus of a lens with a focal length of $2 \mathrm{~m}$. That means, that ionized tracks longer than $1 \mathrm{~m}$ may be initiated with this laser.

\subsection{Space resolution}

The amplifier signal delay - dependent on the electron drift time - was measured with respect to the signal from the photodiode. The time spectrum

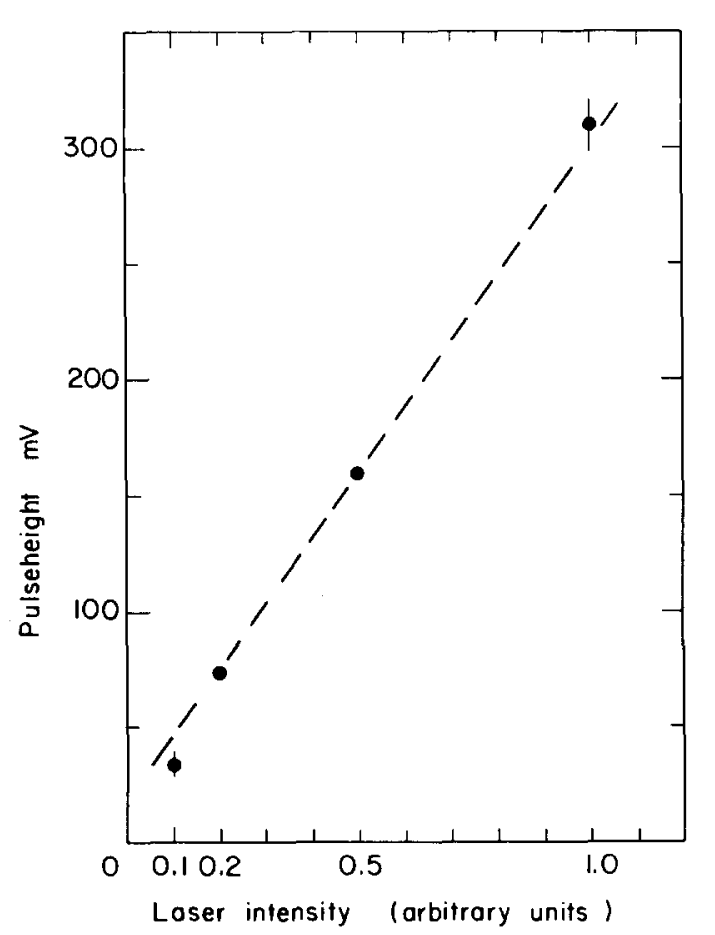

Fig. 2. Maximum pulse height of the ionization signal as a function of the laser intensity. 


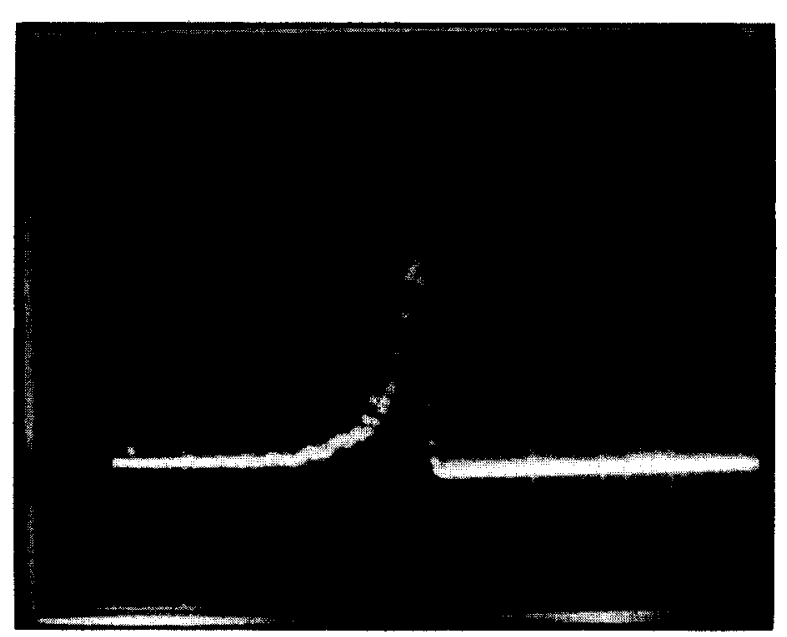

Fig. 3. Pulse height spectrum of the ionization signal ( $\mathrm{fwhm}=$ $9 \%$, including gas amplification spread). The tiny peak indicates the pedestal.

(fig. 4) was obtained in the ethane mixture with the $1 \mathrm{~m}$ focal length, $5 \mathrm{~cm}$ away from the focus. The separation between the first (a) and second (b) peak corresponds to the addition of a 20 ns cable delay, the separation of the second and third peak (c) to an additional $1 \mathrm{~mm}$ drift distance (chamber displaced vertically).

The time spectrum indicates that the boundary of

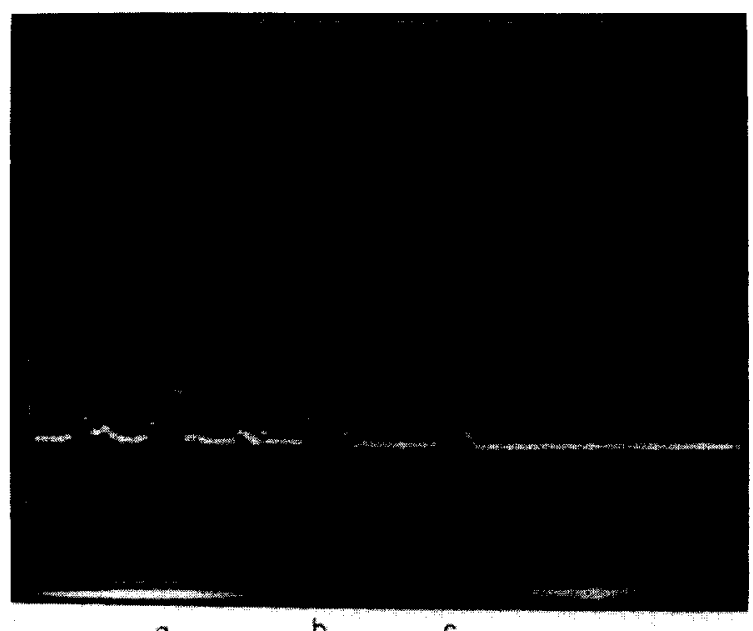

0

w

Fig. 4. Time spectrum of the ionization signal in $\mathrm{Ar} / \mathrm{C}_{2} \mathrm{H}_{6}$ $(\mathrm{f} w h m=2 \mathrm{~ns})$. Delayed trigger from laser pulse. Separation of peaks (a) and (b) caused by 20 ns cable delay, separation of peaks (b) and (c) by an additional $1 \mathrm{~mm}$ drift distance in the gas. the ionized track is defined with a fwhm of 2 ns. Taking into account the measured electron drift velocity, this represents a space resolution of 50 microns rms. As variations from time slewing of the electronics (20 ns risetime) and the instability of the laser beam position and electronics are included in this value, further improvements may be possible.

\subsection{Double tracks}

By replacing the photodiode (fig. 1) by a prism, the laser beam was reflected back in to the drift chamber. Due to the lack of more sophisticated equipment we had to postpone detailed measurements. However, we checked qualitatively the double track technique. Using the $f=1 \mathrm{~m}$ lens, the scope clearly showed two pulses on the same sense wire with a time separation corresponding to the space separation of the two beams. The relative amplitude of the peaks indicated saturation effects, dependent on the separation vertical to and along the wire. As the light path from wire to prism and back was $60 \mathrm{~cm}$, this test again demonstrates the possibility of long tracks.

\section{Applications}

The measured characteristics, important for the application of the laser beam technique, are:

(a) precise localization of the beam $\left(\sigma_{x}=50 \mathrm{mi}\right.$ crons, $\sigma_{\mathrm{t}}<1 \mathrm{~ns}$ ),

(b) negligible scattering,

(c) no deflection in a magnetic field,

(d) narrow pulse height distribution (1/10 of the Landau distribution), and

(e) simple formation of double tracks.

We will briefly discuss only some of the applications to image chamber systems. Most of these we intend to investigate on the Axial Field Spectrometer at the ISR.

\subsection{Surveying}

Usually, surveying of complex imaging chambers is achieved through drift time measurements in particle beams. The laser beam characteristics mentioned [(a)-(c)] are obviously of interest for such measurements. As the laser may easily be pointed in any direction, azimuthal effects of wire displacements (due to the combined action of gravity and electrostatic forces) may also readily be studied. 


\subsection{Measurements in magnetic fields}

The laser beam offers the advantage of providing, in situ, a very stiff beam, precisely localized in time and space, independent of the magnetic field. This permits separate measurement of relative wire positions, electron drift velocity and drift angle in the magnetic field. In data taking with the actual beams, these parameters are all interrelated and their separation is a time consuming iterative process.

\subsection{Amplitude calibration}

For $\mathrm{d} E / \mathrm{d} x$ measurements, variations of gas amplification from wire to wire, as well as along the wires have to be known precisely. For flat geometries movable or multiple radioactive sources may be used for calibration, for cylindrical geometries only particle beam studies in situ are available. In particle beams, it is difficult to obtain sufficient statistics for any point in the chamber, due to large pulse height fluctuations (fwhm $\sim 100 \%$ ), to the interference of neighbouring tracks and corrections from track curvature, angle and distance from the sense wire.

Given sufficient optical access, the laser beam again offers an advantage in being straight, well localized and providing a pulse height spectrum narrower by one order of magnitude.

\subsection{Study of double track effects}

Double track effects are becoming increasingly important for imaging and $\mathrm{d} E / \mathrm{d} x$ chambers (especially for jet research), but little experimental information is available due to technical problems.

With particle beams it is difficult to study adjacent tracks under controlled conditions. Low density beams demand outrageous data taking times; high density beams, serious corrections due to the difficult localization of the two tracks, large pulse height fluctuations and the interference from additional unwanted tracks.

Here, the laser beam offers a unique possibility. Any angle and separation between two tracks may be readily obtained. The narrow pulse height spectrum demands only low statistics. Various particle momenta, gas densities and pulse heights may be faked by changing the light intensity in one or both beams.

Other applications based on the good track localization in space and time and the variable density of primary ionization include precision measurements on saturation effects, drift velocities, lateral and longitudinal diffusion constants.

The author would like to thank especially Mr. Brunet for the skilful construction of the laser and the drift chambers, Dr. Geissler and Dr. Neracher for many discussions and for their help with laser problems and for providing auxiliary equipment and $\mathrm{Mr}$. Schuler for the preparation of related electronics. Continuous support for the project from Dr. Lazeyras and Dr. Minten is gratefully acknowledged.

\section{References}

[1] A. Gold et al., Phys. Rev. Lett. 14 (1965) 60; G.A. Askaryan et al., JETP 21 (1965) 190.

[2] J. Reintjes et al., JEEE, QE 14 (1978) 581.

[3] R.G. Meyerand et al., Phys. Rev. Lett. 11 (1963) 401.

[4] R.G. Tomlinson, Phys. Rev. Lett. 14 (1965) 489.

[5] D.H. Gill et al., Phys. Rev. Lett. 15 (1965) 845.

[6] H.T. Buscher et al., Phys. Rev. Lett. 15 (1965) 847.

[7] V.E. Mitsuk, XIII Int. Conf. on: Phenomena in ionized gases (1967) p. 260.

[8] A.A. Dougal et al., XIII Int. Conf. on Phenomena in ionized gases (1967) p. 262.

[9] J. Bourotte and B. Sadoulet, private communication, submitted to Nucl. Instr. and Meth.

[10] MOPA-1000 from Multilasers, Geneva, Switzerland.

[11] Data sheets from Hewlett-Packard for HPZ-4220. 